\title{
The learning of basic-level categories by pigeons: The prototype effect, attention, and effects of categorization
}

\author{
Masako Jitsumori • Midori Ohkita • Tomokazu Ushitani
}

Published online: 12 April 2011

(C) Psychonomic Society, Inc. 2011

\begin{abstract}
Pigeons were trained to classify composite faces of two categories created by mimicking the structure of basic-level categories, with each face consisting of an itemspecific component and a common component diagnostic for its category. Classification accuracy increased as the proportion of common components increased, regardless of familiar and novel item-specific components, with the best discrimination occurring at untrained original faces used as the common components. A no-categorization control condition suggested that categorization gives rise to equivalence for item-specific components and distinctiveness for degrees of prototypicality. When some item-specific components were shared by exemplars of the contrasting categories, those that were not overlapping between the categories became the effective cues for the pigeons' responses. Implications of these results are discussed in the context of current categorization and associative learning theories.
\end{abstract}

Keywords Categorization - Prototype effect - Attention . Acquired equivalence $\cdot$ Acquired distinctiveness .

Morphing · Pigeons

\section{Introduction}

Most natural categories are not defined by singly necessary and jointly sufficient attributes. However, attributes tend to be correlated with each other, either positively or negatively;

M. Jitsumori $(\bowtie) \cdot$ M. Ohkita $\cdot$ T. Ushitani

Department of Cognitive and Information

Sciences, Faculty of Letters, Chiba University,

1-33 Yayoicho, Inage-ku,

Chiba 263-8522, Japan

e-mail: mjitsu@L.chiba-u.ac.jp the presence of attribute A tends to be associated with the presence of attribute $\mathrm{B}$ and with the absence of attribute $\mathrm{C}$ (Taylor, 1995). The correlation of attributes keeps the categories discrete, so that each has a well-structured family resemblance. Family resemblance allows the most prototypical members to have the highest resemblance to the other members of the category and the least resemblance to the members of other categories. Rosch has argued that representativeness within a category and distinctiveness from contrast categories are correlated with prototypicality in natural categories (Rosch, 1978; Rosch \& Mervis, 1975).

To study the learning of categories that are not definable by singly necessary and jointly sufficient attributes, investigators have often constructed a simple form of artificial categories consisting of binary-valued feature dimensions (BVDs). For example, one category might consist of the exemplars $(1111,1110,1101,1011,0111)$ and the other category of the exemplars $(0000,0001,0010,0100,1000)$, with the logical values 1 and 0 being binary-valued features on each of the dimensions that are independent of one another. The exemplars having the feature value 1 or 0 on all the dimensions (1111 and 0000) are referred to as prototypes. It has been generally found that animals, such as pigeons and monkeys, successfully learn to discriminate the BVD categories, with the best discrimination occurring at the prototypes, during acquisition training or to classify untrained prototypes as accurately as, or even better than, trained exemplars during generalization testing (in pigeons, Aydin \& Pearce, 1994; Huber \& Lenz, 1993; Jitsumori, 1993, 1996; Lea \& Harrison, 1978; Lea, Lohman, \& Ryan 1993; Lea \& Ryan, 1983; von Fersen \& Lea, 1990; in rhesus monkeys, Jitsumori, 1994; in baboons, Dépy, Fagot, \& Vauclair, 1997).

The advantages of the prototypes of BVD categories confirm the principles of family resemblance; the prototype 
of one category has the highest resemblance to the other members of the category and the least resemblance to the members of the contrasting category. Actually, the category members are created by the least number of transformations of the binary-valued features from the prototype (one dimension at a time). However, the prototype of one category is also the member of that category furthest away from the prototype of the other on a feature-sum dimension (in the example, four vs. three), and it has none of the features possessed by exemplars of the opposite category (1111 has no zeros in it, and 0000 has no ones). Researchers often have argued that the prototype advantage shown by animals during generalization testing is confounded with peak shift (i.e., better performance on unseen exemplars more extreme than old training exemplars) occurring on a stimulus dimension along which exemplars from contrasting categories are arranged in a linear fashion (e.g., Huber \& Lenz, 1993; Jitsumori, 1996; Lea \& Harrison, 1978; McLaren, Bennett, Guttman-Nahir, Kim, \& Mackintosh, 1995; see also Mackintosh, 1995, for the distance-from-category-boundary peak shift). Peak shift was first demonstrated by Hanson (1959) in pigeons and has since been shown with various physical dimensions, such as wavelength, brightness, vertical orientation, and so forth, in many different species, including humans (see Ghirlanda \& Enquist, 2003, for a review).

Our aim is to investigate a central tendency effect with the artificial categories created by mimicking the structure of basic-level categories. At a basic level of categorization (e.g., chair), perceptual, as well as functional, attributes are shared by all or most members of the category, but these attributes are different from those of contrasting categories (e.g., table, bed, and hutch). At this basic level, objects in a category look very much like one another, whereas objects in different categories are perceptually distinct. Categories below the basic level are subordinate categories. Members of a subordinate category (e.g., kitchen chair) share a large number of attributes, but these attributes are also shared with members of contrasting categories at the same level (e.g., diningroom chair, dentist's chair), so that the category is not maximally distinct from other categories. At the superordinate level of categorization (e.g., furniture), members of a category share only a few attributes. Basic-level categories are most inclusive and best mirror the correlational structures existing in the real world (Rosch, 1978; see also Corter \& Gluck, 1992).

It also has been generally agreed that instances of natural categories have not only the aspects that determine membership in the category (e.g., birds have wings), but also the aspects that determine individual identities (e.g., wings of an eagle are distinct from those of a robin). That is, the exact realization of an attribute such as winged varies from instance to instance (Medin, Dewey, \& Murphy
1983). In contrast, the individual exemplars of BVD categories are created using different combinations of the binary values possessed by the prototypes, and thus there is no feature unique to a particular member. Medin et al. claimed that studies using artificially constructed categories appear to have a deficiency in the idiosyncratic information of natural objects.

Before describing our specific hypotheses, we first discuss the research strategy that we followed in constructing the stimuli and forming the categories. Figure 1 shows the categories created by morphing human faces. We used human faces because (1) they are naturalistic stimuli, (2) they have a richness of details and are potentially discriminable along multiple dimensions, (3) they have been used successfully for pigeons in the previous categorization studies (e.g., Jitsumori \& Yoshihara, 1997; Troje, Huber, Loidolt, Aust, \& Fieder 1999), (4) pigeons are naïve with respect to human-face categories and may perform on the basis of purely visual cues, and (5) pigeons perceive morphed faces as being similar to, but still discriminably different from, the originals from which they were created (Makino \& Jitsumori, 2000; see also Jitsumori, Shimada, \& Inoue, 2006).

Faces A, B, C, and D in category 1 and faces E, F, G, and $H$ in category 2 are Japanese male students arbitrarily assigned to the categories. Each face was morphed with a single unique face ( $\mathrm{M}$ in category 1 and $\mathrm{N}$ in category 2 ) to create category members (AM-DM in category 1 and EN$\mathrm{HN}$ in category 2). We also created $50 \%$ morphs of the six possible pairs of $\mathrm{A}, \mathrm{B}, \mathrm{C}$, and $\mathrm{D}(\mathrm{AB}, \mathrm{AC}, \mathrm{AD}, \mathrm{BC}, \mathrm{BD}$, and $\mathrm{CD}$ ), each of which was then morphed with $\mathrm{M}$ to create additional composite faces in category 1. Similarly, six additional morphs were created for category 2 . We used a Caucasian male student as $\mathrm{M}$ and a Japanese male professor as N. So category 1 is a Caucasian face class characterized by the common component $\mathrm{M}$, and Category 2 is an Asian face class characterized by the common component $\mathrm{N}$. Faces $\mathrm{A}-\mathrm{D}$ and faces $\mathrm{E}-\mathrm{H}$ are the item-specific, idiosyncratic components that differ from instance to instance within and also between the categories. In this way, we created two artificial categories that consisted of exemplars that differed in idiosyncratic details but that each had a well-structured family resemblance.

The morphed faces connected with broken lines in Fig. 1 have the same proportion (35\%) of the common component (35\% M faces in category $1,35 \% \mathrm{~N}$ faces in category 2$)$. The morphing process is a two-step process involving warping and averaging on a pixel-by-pixel basis (see Busey \& Tunnicliff, 1999, for more details). We do not know the exact physical properties created by morphing, and we are unable to describe the specific features available for pigeons to discriminate the morphed images. We still consider the proportion of the common component to be a 
Fig. 1 Grayscale reproductions of the exemplars. In category 1 , the $\mathrm{A}, \mathrm{B}, \mathrm{C}$, and $\mathrm{D}$ faces and their $50 \%$ morphs $(\mathrm{AB}, \mathrm{AC}$, $\mathrm{AD}, \mathrm{BC}, \mathrm{BD}$, and $\mathrm{CD}$ ) were morphed with the $M$ face (AD, $\mathrm{BC}$, and those created by blending each of these faces with $\mathrm{M}$ are not shown). In category 2 , the $\mathrm{E}, \mathrm{F}, \mathrm{G}$, and $\mathrm{H}$ faces and their $50 \%$ morphs (EF, EG, EH, FG, FH, and $\mathrm{GH}$ ) were morphed with the $\mathrm{N}$ face $(\mathrm{EH}, \mathrm{FG}$, and those created by blending each of these faces with $\mathrm{N}$ are not shown)
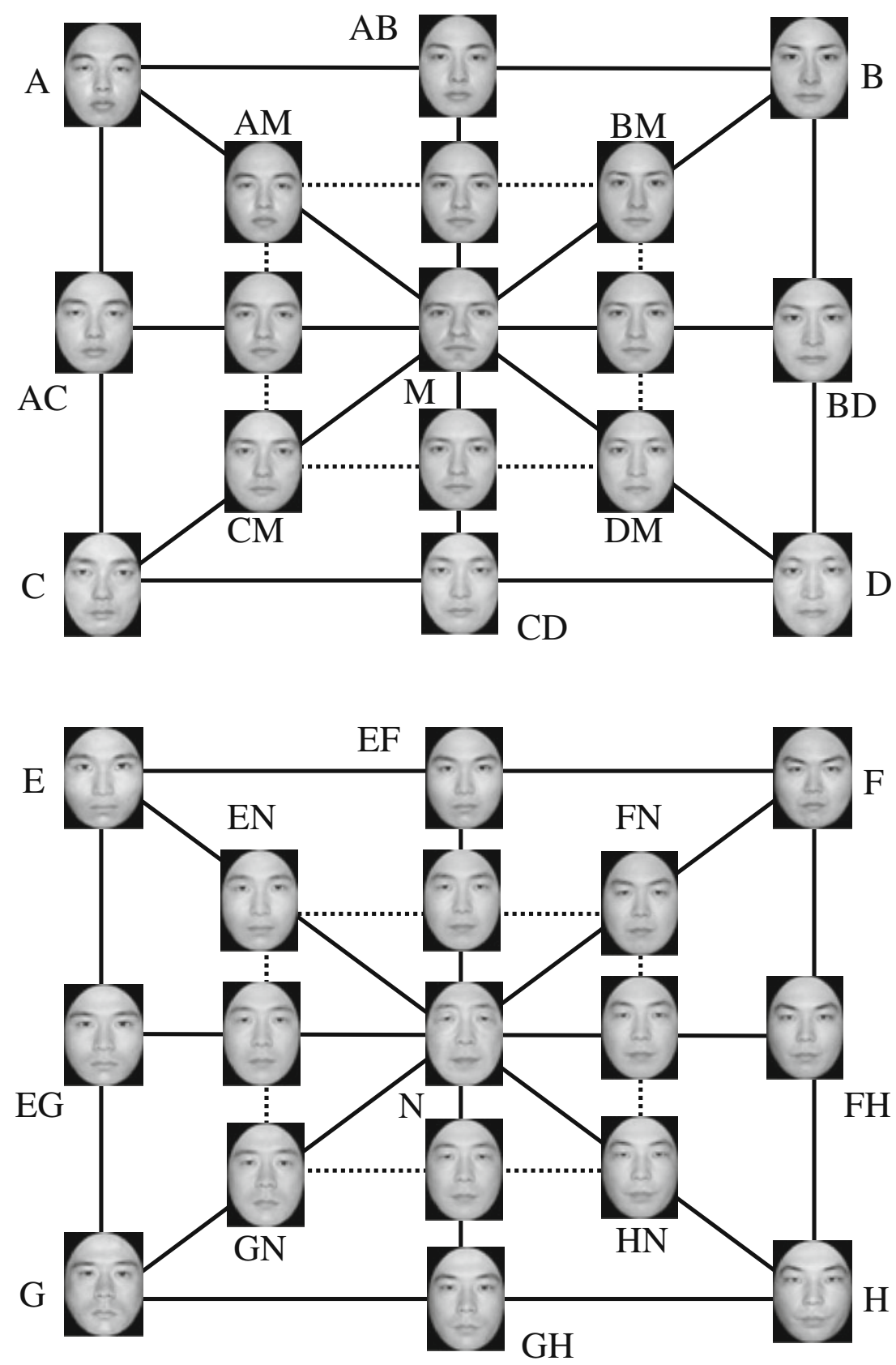

psychological dimension despite the difficulty in associating it with simple, physically measurable properties (see also Corneille \& Judd, 1999).

Various exemplars that differ in degree of typicality are on the lines that join the common component face (face $\mathrm{M}$ in category 1 , face $\mathrm{N}$ in category 2 ) with the idiosyncratic component faces. We defined faces $\mathrm{M}$ and $\mathrm{N}$ as the prototypes of the categories. The prototype represents the category by emphasizing features of the category that distinguish it from the other category. The categories permit membership to a large, potentially infinite number of instances that have a wide variety of novel idiosyncratic components other than those used to create the exemplars shown in Fig. 1. Therefore, the categories are ill-defined in the same way that open-ended natural categories are.

Note that the common component of one category is not shared by exemplars of the other category; the presence of the common component $\mathrm{M}$ is associated with the absence of the common component $\mathrm{N}$, and vice versa. The common component features of the discrete categories do not constitute a continuous feature dimension, so that having more of the features distinctive to one category (say, those of $\mathrm{M}$ in category 1) does not lead to having fewer of the features distinctive to the alternative category (say, those of $\mathrm{N}$ in category 2). Accordingly, peak shift will not occur (for peak shift, see Blough, 1975; Mackintosh, 1995; Spence, 
1937; for a caricature effect on a single face morph dimension in humans, see Corneille, Goldstone, Queller, \& Potter, 2006; Goldstone, Steyvers, \& Rogosky, 2003).

In Experiment 1, we trained pigeons, in a go/no-go discrimination procedure, to discriminate exemplars of one category from those of the other category. Not only the common components, but also the idiosyncratic components allowed classification of the training exemplars. A question of interest is whether classification accuracy increases as a function of the proportion of common components, with the best discrimination occurring at the untrained prototypes. An additional interest is whether and to what extent the pigeons transfer the discrimination to novel stimuli that possess new idiosyncratic components. Experiment 2 was conducted as a control for Experiment 1, in which new pigeons were trained to discriminate just a single positive exemplar from a single negative exemplar arbitrarily selected from those used in Experiment 1. In Experiments 3 and 4, we used the categories similar to those in Experiment 1, but some idiosyncratic components were overlapping between the categories and others were not. Experiment 3 explored effects of this treatment on categorization, and Experiment 4 examined whether pigeons made use of the idiosyncratic features that were not overlapping between the categories.

In these experiments, responding to the positive stimuli was reinforced by a variable interval (VI) schedule during training. Subsequent generalization testing was carried out in extinction. Namely, reinforced trials using training stimuli were not introduced to maintain overall responding during the testing period. A serious disadvantage of the maintained procedure is that discrimination between training and test stimuli is acquired, so that responding to stimuli highly discriminable from the training stimuli fall rapidly to an uninformative zero level. It has been shown that the maintained procedure is useful when closely spaced stimuli are tested (e.g., Blough, 1972), allowing us to obtain steep gradients over a narrow range of stimuli. For comparing response probability over a broad stimulus range, on the other hand, the method of obtaining data in the course of extinction is more appropriate (Blough, 1972; Rilling, 1977). It is impossible to determine, in advance, the number or proportion of training trials that is enough to maintain overall responding but does not largely or significantly change the shape and slope of the generalization gradient in each experiment. Specifically, because the present study was concerned with whether novel exemplars would be classified better than the old training exemplars, the maintained procedure would make our findings difficult to interpret. Therefore, we used the extinction procedure. Of course, repeated exposures to extinction reduce key pecking. However, the decrease in response rate during extinction is gradual after VI reinforcement, and the differences due to the slow decrease are averaged out by testing different stimuli in a randomized blocks design.We obtained the total number of responses made by each subject to each stimulus during generalization testing, on the basis of which relative response rates were calculated to obtain a generalization gradient averaged among subjects. As will be noted in each experiment, random sequences of display times were programmed independently of the randomized presentations, so that the total exposure time to each stimulus during generalization testing was to be the same for each subject.

\section{Experiment 1}

We prepared two groups of pigeons (Group $35+35$ - and Group $35+50-)$. The positive and negative roles of the categories were counterbalanced among pigeons in each group. Hereafter, we will refer to the prototypes (faces $M$ and $\mathrm{N}$ ) as $\mathrm{P}+$ in the positive category and $\mathrm{P}$ - in the negative category, regardless of the positive and negative roles of categories 1 and 2 . The positive training exemplars were the same for the two groups $(35 \% \mathrm{P}+)$, while the negative training exemplars differed between the groups $(35 \% \mathrm{P}$ - for Group $35+35$ - and 50\%P- for Group $35+50-$ ). After training, the pigeons were tested for transfer to novel morphs on the positive and negative face-morph dimensions.

In one test, referred to as the familiar idiosyncratic component (FIC), the idiosyncratic components are the same as those of the training stimulus sets. In the other test, referred to as the novel idiosyncratic component (NIC), they are novel. A critical question is whether classification accuracy increases as a function of the $\mathrm{P}$ proportion, regardless of the familiar or novel idiosyncratic components. Note that the prototype represents the regularity of exemplars in its category but is not an average of the training exemplars. For example, a physical average of the composite faces that contain $35 \% \mathrm{P}(65 \% \mathrm{~A}, 65 \% \mathrm{~B}, 65 \% \mathrm{C}$, or $65 \% \mathrm{D})$ is $35 \% \mathrm{P}(65 \% \mathrm{ABCD}$; $\mathrm{ABCD}$ is a $25 \%$ morph of $\mathrm{A}, \mathrm{B}, \mathrm{C}$, and $\mathrm{D})$. The average varies depending on the idiosyncratic component faces and the proportion of the common component used to create the training stimulus set, while the prototype is invariant.

If pigeons learned the individual stimulus-response relations by rote, the generalization gradient in the FIC test would have a peak at the training exemplars, and transfer to the stimuli that have novel idiosyncratic components in the NIC test would be largely restricted. As we described earlier, peak shift may not occur in the FIC test. On the other hand, if the pigeons learned to classify the stimuli so as to maximize within-categories similarity and betweencategories dissimilarity, generalization gradients would increase as a function of the $\mathrm{P}+$ proportion and decrease 
as a function of the P-proportion, regardless of the familiar or novel idiosyncratic components. Then, we may consider that a prototype effect that is free from peak shift was obtained.

An additional question is whether and to what extent the pigeons would make use of the idiosyncratic components to learn the categories. One can predict that, to refrain from pecking to the individual negative training stimuli, Group $35+$ 35- would make use of the idiosyncratic component features more effectively than Group $35+50$ - would, simply because the negative stimuli for the former group have a larger proportion of the idiosyncratic components. A question of interest is whether, in the FIC test, Group $35+35$ - would discriminate the negative idiosyncratic component faces from the positive ones more accurately than would Group $35+50$ -

\section{Method}

Animals Eight experimentally naïve pigeons, kept at $85 \%$ of their free-feeding body weights, were arbitrarily divided into two groups (Group $35+35$ - and Group $35+50-$ ). Water and grit were freely available in their individual home cages.

Apparatus The experiment used two identical operant conditioning chambers $(35 \times 35 \times 35 \mathrm{~cm})$. All of the stimuli were presented on TFT color monitors (Sony, PVM$6041 \mathrm{Q})$, visible through a viewing window $(10 \times 6 \mathrm{~cm})$ located in the middle of an aluminum front panel of each chamber. The bottom edge of the viewing window was $20 \mathrm{~cm}$ above the chamber floor. The monitor was located $1.5 \mathrm{~cm}$ behind an infrared touch frame (Carroll Touch, Unitouch). A food aperture $(1.5 \times 4 \mathrm{~cm})$, located on the floor and centered below the viewing window, afforded pigeons access to a solenoid-operated food tray containing a mixture of grains. A houselight $(3 \mathrm{~W})$ in the center of the ceiling dimly illuminated the chamber.

The chambers and the video monitors were located in a darkened testing room. Computer programs driving the presentation of stimuli and controlling the feeder were developed in Visual Basic 6.0 (Microsoft).

Stimulus materials Frontal views of 14 human faces (12 Japanese male students, 1 Caucasian male student, and 1 Japanese male professor) with neutral expressions were taken in color with a digital camera (Olympus, C-730) under the same lighting condition. The inner faces were cut into an ellipse of the same size, and the background was painted black using Photoshop (Adobe). The height and width of the stimuli were $25 \mathrm{~mm}$ (102 pixels) and $18 \mathrm{~mm}$ (77 pixels) when they were displayed on the color monitor. The Caucasian student's blue eyes were digitally recolored to dark brown.
Eight faces arbitrarily selected from the faces of 12 Japanese male students served as the idiosyncratic components of the positive and negative training exemplars. The remaining four faces served as the novel idiosyncratic components. The faces were arbitrarily assigned to the two categories. Blending was conducted by commercially available morphing software (Morph, Gryphon), as described above.

Figure 2 shows the test stimuli in category 1 (those in category 2 are not shown). The stimulus set used for the FIC test consisted of morphs ranging from each idiosyncratic component face to the prototype. Seven faces $(\mathrm{P} \%=$ $0,10,20,35,50,70$, and 100) were prepared on each facemorph dimension. There were 28 faces $(7$ faces $\times 4$ dimensions) in each category, for a total of 56 stimuli (including four identical positive or negative prototypes). Morphs between an average of 2 idiosyncratic component faces $(\mathrm{AB}, \mathrm{AC}, \mathrm{AD}, \mathrm{BC}, \mathrm{BD}$, or $\mathrm{CD}$ in category 1 and the corresponding composite face in category 2) and the prototype in each category were not tested, partly because this would have generated an inappropriately large number of stimuli for generalization testing in extinction, and partly because these morphs were not likely to add specific information. The stimulus set used for the NIC test

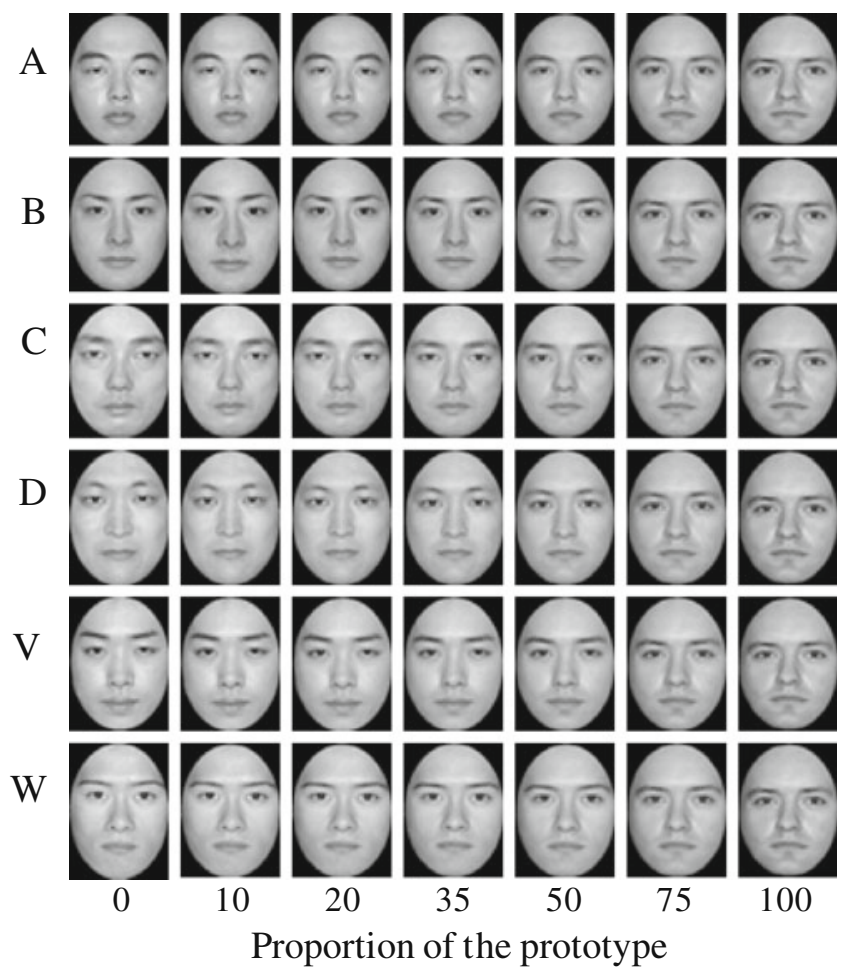

Fig. 2 Grayscale reproductions of the faces in category 1 used for generalization testing. All of the faces in the rightmost column are identical and depict the prototype (the $M$ face in Fig. 1); the test stimuli are created by blending the prototype with each of the leftmost faces, $\mathrm{A}, \mathrm{B}, \mathrm{C}, \mathrm{D}, \mathrm{V}$, and $\mathrm{W}$. The $\mathrm{V}$ and $\mathrm{W}$ faces are the novel faces, not used to create the training exemplars 
consisted of morphs ranging from each of the new idiosyncratic component faces (faces $\mathrm{V}$ and $\mathrm{W}$ in category 1 and two other new faces in category 2) to the prototype, a total of 28 stimuli (including two identical positive or negative prototypes).

Procedure Preliminary training involved magazine training, hand shaping, and reinforcing pecks to a white oval (the same size as the face stimuli) on a fixed ratio 30 schedule. The pigeons were then transferred to a standard go/no-go procedure (see, e.g., Vaughan \& Greene, 1984). A trial began with the presentation of a stimulus in the center of the computer display. A pigeon's pecks to the stimulus were recorded for $10 \mathrm{~s}$ but were not rewarded. Following this period, a VI of $10 \mathrm{~s}$ began (range, 0-20 s; step, $2 \mathrm{~s}$ ). On positive trials, a peck at the end of a scheduled interval was reinforced. On negative trials, the pecks were not reinforced. A negative trial terminated after a scheduled interval had expired and $5 \mathrm{~s}$ had passed without a response. The number of responses during the first $10 \mathrm{~s}$ was used to calculate the response rate. Each trial was followed by a 5-s intertrial interval in which a blank frame was displayed on the monitor.

Each of the 20 training stimuli (10 positives and 10 negatives) was displayed once in a randomized block of 20 trials. A session consisted of three blocks - that is, a total of 60 trials per session. Each day, the order of stimulus presentation was randomized, with the restriction that no more than three successive positive or negative stimuli could occur. Each pigeon received a minimum of 14 sessions, and they continued until $80 \%$ or more of the total responses occurred on positive trials in each of two consecutive daily sessions. Then the pigeons received two test sessions: one FIC test session and one NIC test session. Test sessions were given in extinction (without rewards and without penalties). Two pigeons in each group received the FIC test first and then the NIC test. The opposite was true for the remaining 2 pigeons in the group. Then they received retraining (for a minimum of seven sessions) until the $80 \%$ criterion was regained, which was followed by two test sessions with the order of tests reversed (individually by pigeon). A total of four test sessions was given.

In the FIC test, each of the 56 stimuli ( 28 positives and 28 negatives) was displayed once in a session, with 7 positive and 7 negative stimuli containing different proportions of $\mathrm{P}$ presented once in a randomized block of 14 trials (four blocks per session). In the NIC test, each of the 28 stimuli (14 positives and 14 negatives) was displayed twice in a session, once in the first or second block and once in the third or fourth block. The average presentation time was $20 \mathrm{~s}$, varying from 12 to $28 \mathrm{~s}$. In each test, random sequences of display times were programmed independently of the randomized presentations, under the restriction that the total display time for the stimuli with a given morph proportion of $\mathrm{P}$ was $160 \mathrm{~s}$ over two sessions ( $80 \mathrm{~s}$ in each). The display times were determined (individually by pigeon), so that each individual stimulus was presented for a total of $40 \mathrm{~s}$ over two sessions of the FIC test and a total of $80 \mathrm{~s}$ over two sessions of the NIC test. For example, in a case where the 4 stimuli of a given morph proportion of $\mathrm{P}$ were presented for $12,16,24$, and $28 \mathrm{~s}$ in the first session of the FIC test (display times and their sequences were randomized under the restriction described above), they were presented for 28,24 , 16 , and $12 \mathrm{~s}$, respectively, in the second session (only sequences of stimulus presentations were randomized). The total number of responses over the sessions was subjected to analysis.

Results

Training The pigeons learned the discrimination quickly. The number of sessions required to reach the $80 \%$ criterion for two consecutive days averaged 12.0 (range: 7-15) and 8.3 (range: $5-10$ ) for Group $35+35$ - and Group $35+50$-, respectively. A significant group difference in the number of required sessions was not found, $\mathrm{F}(1,7)=3.08, p=.129$. In this and all other tests, an alpha level of .05 was used.

As we described earlier, training continued for a minimum of 14 sessions. In each session, a discrimination ratio (DR) for the positive $(\mathrm{S}+\mathrm{s})$ and negative (S-s) stimuli was calculated for each bird, by dividing the responses to $\mathrm{S}+\mathrm{s}$ by the total responses to $\mathrm{S}+\mathrm{s}$ and $\mathrm{S}-\mathrm{s}$. The mean DR was slightly, but consistently, larger in Group $35+50$ - than Group $35+35$ - over the sessions (the data are not shown). However, a two-way repeated measures analysis of variance (ANOVA), with group as a between-subjects variable and day as a withinsubjects variable, failed to show a significant effect of group, $\mathrm{F}(1,6)=2.30, p=.181$. The main effect of day was significant, $\mathrm{F}(13,78)=25.99, p<.001$. The interaction was not significant, $\mathrm{F}(13,78)=0.65, p=.809$.

Generalization testing The average number of responses in Group 35 + 35- was 959.5 (range: 692-1,407) and 880.8 (range: 512-1,173) in the FIC and NIC tests, respectively. In Group $35+50$-, these numbers were 1,121.0 (range: 879-1,257) and 1,245.7 (range: 607-2,275). Individual pigeons differed widely in their rate of pecking. We calculated for each pigeon relative responses to the different sets of test stimuli as a proportion of the total number of responses occurring to all the stimuli in each test, from which we obtained an average generalization gradient.

Figure 3 separately shows the mean relative responses for the two tests for each group, plotted as a function of the $\mathrm{P}$ proportion. The abscissa ranges from -100 (the negative prototype) to 0 (the positive and negative idiosyncratic component faces), then further to 100 (the positive 

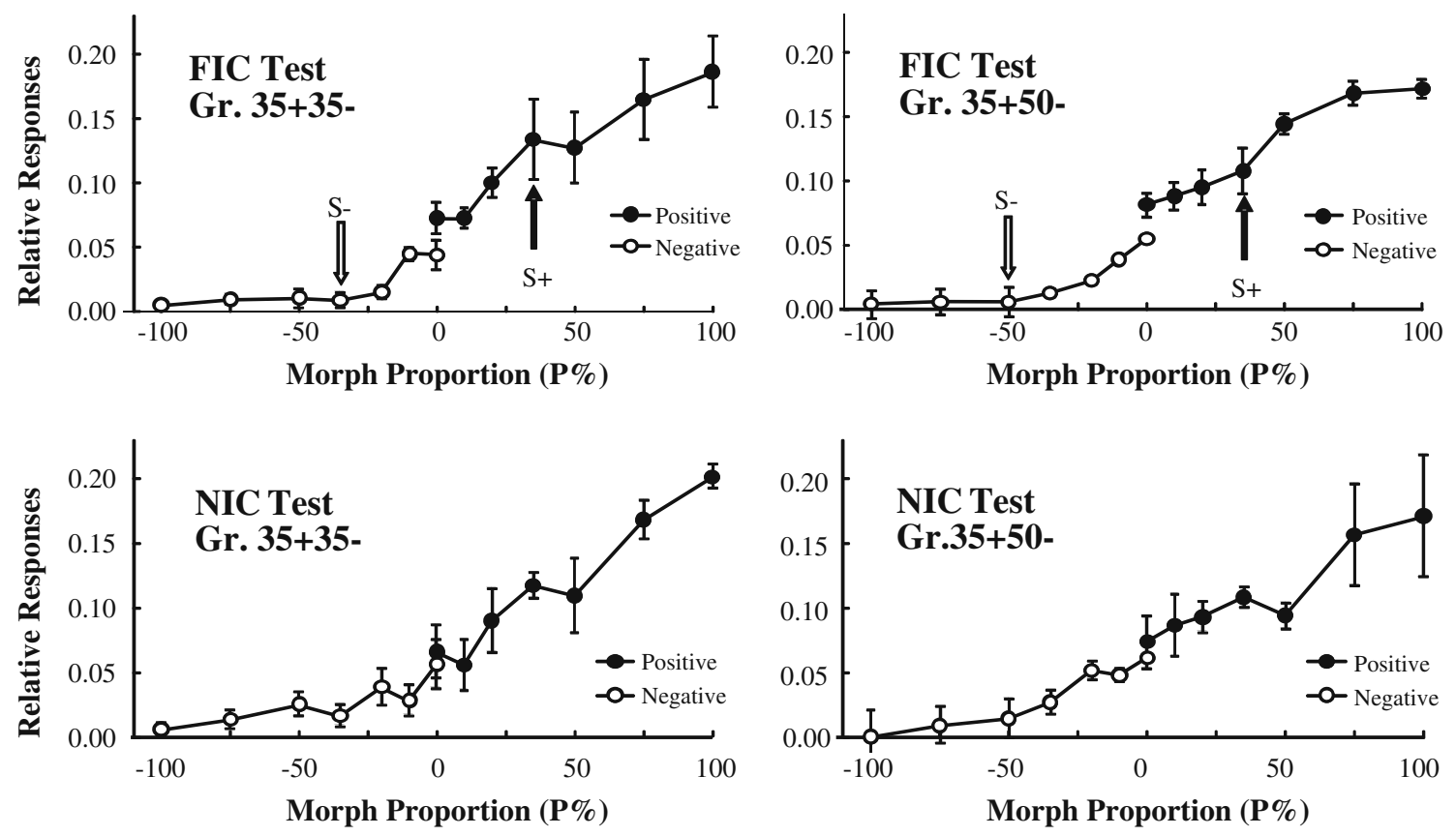

Fig. 3 Mean relative responses to the positive and negative test stimuli as a function of the proportion of the common component in the familiar idiosyncratic component (FIC) test (upper panels) and the novel idiosyncratic component (NIC) test (lower panels) in Experiment 1.

prototype). The effect of morph proportion was striking. The positive generalization gradient generally increased as a function of the $\mathrm{P}+$ proportion, with the highest rate of responses at the positive prototype. The negative generalization gradient was relatively shallow but tended to decrease as a function of the P-proportion, with the lowest rate of responses at the negative prototype.

A three-way repeated measures ANOVA was separately conducted on the data for the positive and negative test stimuli, with group (Group $35+35$ - vs. Group $35+50$-) as a between-subjects variable and test (FIC vs. NIC) and morph proportion ( $0 \%$ vs. $10 \%$ vs. $20 \%$ vs. $35 \%$ vs. $50 \%$ vs. $75 \%$ vs. $100 \%$ ) as within-subjects variables. Separate statistical analyses were carried out in this and all other cases, because response rates to negative stimuli were almost certain to be lower and to have less absolute variability than those to positive stimuli and, therefore, combined analyses are likely to be very difficult to interpret. For the positive test stimuli, the effect of morph proportion was significant, $\mathrm{F}(6,36)=13.91, p<.001$. The effects of group, $\mathrm{F}(1,6)=0.07, p=.798$, and test, $\mathrm{F}(1,6)=$ $2.87, p=.142$, the interactions between any two variables, and the three-way interaction were all not significant. For the negative test stimuli, the effect of morph proportion was significant, $\mathrm{F}(6,36)=22.83, p<.001$. The effects of group, $\mathrm{F}(1,6)=0.07, p=.798$, and test, $\mathrm{F}(1,6)=2.97, p=.136$, the interactions between any two variables, and the threeway interaction were all not significant. These findings
The error bars show standard errors. The white and black arrows indicate which datapoints were observed for the negative (S-) and positive $(\mathrm{S}+)$ sets of training stimuli, respectively

confirmed that morph proportion was the controlling factor in the pigeons' responses, regardless of the familiar or novel idiosyncratic components of the stimuli.

We hoped to see which group would more accurately discriminate the familiar positive and negative idiosyncratic component faces $(\mathrm{P} \%=0)$ in the FIC test, but there was no notable group difference. The two groups of pigeons showed slightly, but significantly, higher rates of responses, $\mathrm{t}(7)=2.82, p=.026$ (two-tailed paired $\mathrm{t}$ test), to the positive idiosyncratic component faces than to the negative ones. Such an effect was naturally not found in the NIC test. The mean DRs calculated by dividing the responses to the positive stimuli by the total responses to the positive and negative stimuli in the FIC test were .85 and .86 for Group $35+35$ - and Group $35+50-$, respectively. Those in the NIC test were .79 and .79 for Group $35+35$ - and Group $35+50$-, respectively. These findings suggest that the idiosyncratic components did have a small but clear impact on discrimination.

\section{Discussion}

A prototype effect was observed. As we discussed earlier, we may consider that the effect is free from peak shift. Note also that, unlike artificial categories consisting of binaryvalued features, the composite faces take new values as a result of morphing. The better performance we observed on unseen exemplars, as compared with old training exemplars, cannot be accounted for by an additive integration of 
learned features (e.g., the multiple feature model of Lea \& Ryan, 1983; see also Huber \& Lenz, 1993; Jitsumori \& Yoshihara, 1997). A question then arises as to why the pigeons trained with the composite faces containing only $35 \% \mathrm{P}$ or $50 \% \mathrm{P}$ then best discriminated the untrained faces containing $100 \% \mathrm{P}$.

We may assume that a composite face is represented by an array of features, some of which are similar to those of other composite face(s). Perhaps the features are not independent but interact to influence each other's activation strength. The more the composite faces that possess features similar to a given feature, the higher activation that feature will acquire by training. This interactive aspect of features helps to explain the prototype effect, by assuming that the number of features similar to the highly activated features increases as the proportion of the common component increases.

However, we are unable to specify the exact features used by the pigeons to categorize the composite faces in the present study, and we know even less about the similarity relations among such features. Recently, Soto and Wasserman (2010) proposed a common elements model, in which complex natural stimuli are represented by arrays of hypothetical elements (microfeatures). Some elements can be common to all or most members of a category (the category-specific elements), whereas others can be common to few members or belong to just one member (the stimulus-specific elements). Exemplars of categories are represented in terms of the specificity distribution, which determines the proportion of elements in varied levels of specificity. The model will predict the prototype effect shown by the pigeons in the present experiment, ignoring the similarity relations among features, stimuli, and categories but simply assuming that the proportion of the hypothetical category-specific elements increases as a function of the proportion of the common component.

However, our feature-learning-and-generalization account, as well as Soto and Wasserman's model, may have considerable difficulty explaining the generalization gradient in the NIC test. Blending a new idiosyncratic component face into the common component face produces features physically different from those in the training stimulus set, and when the proportion of the common component decreases, features dissimilar to the learned features would increase rapidly. Then, the generalization gradient in the NIC test should be steeper than the generalization gradient in the FIC test (see also Soto \& Wasserman, 2010, for the prediction of generalization decrement to novel exemplars). However, comparisons between the upper and lower panels in Fig. 3 revealed no notable difference. One may argue that the generalization gradient obtained in the NIC test can be explained in terms of familiarity/novelty judgment; novelty increases as the proportion of the novel idiosyncratic component increases, while familiarity increases as the proportion of the common component increases. Such a familiarity/novelty account, however, does not explain the results of the FIC test, in which the pigeons showed the best discrimination for the novel prototypes, rather than for the familiar training exemplars.

These considerations required us to examine the effect of categorization training on generalization to the test stimuli. Experiment 2 was designed as a no-categorization control condition to examine this issue.

\section{Experiment 2}

We trained new pigeons to discriminate just one positive stimulus from one negative stimulus selected from those used in Experiment 1. More specifically, 4 pigeons were trained with one of the following four pairs of stimuli: the $\mathrm{AM}$ and $\mathrm{EN}, \mathrm{BM}$ and $\mathrm{FN}, \mathrm{CM}$ and $\mathrm{GN}$, and $\mathrm{DM}$ and $\mathrm{HN}$ faces, shown in Fig. 1 (the proportion of the common component was $35 \%$ ). The positive and negative roles of the stimulus sets were counterbalanced among the 4 pigeons.

The pigeons were then tested for transfer to the novel stimuli used in the generalization tests in Experiment 1. The generalization tests corresponding to the FIC and NIC tests in Experiment 1 will be referred to as the familiar component (FC) and novel component(NC) tests, respectively.

\section{Method}

Animals, apparatus, and stimulus materials Four new pigeons served as subjects. They had previously served in tasks using the touch screen but had no prior experience with pictorial stimuli. Housing, maintenance, apparatus, and stimulus materials were the same as those in Experiment 1.

Procedure One positive stimulus and one negative stimulus were displayed 30 times in a session of 60 trials. Each pigeon received a minimum of 12 sessions, and they continued until $80 \%$ or more of the total responses occurred in the positive trials in each of two consecutive daily sessions.

After completion of the discrimination training, the pigeons received four test sessions: two sessions in the FC test and two sessions in the NC test. The order of test sessions was counterbalanced among the 4 pigeons, as in Experiment 1. Retraining intervened as in Experiment 1, which continued (for a minimum of seven sessions) until the $80 \%$ criterion was regained.

The faces presented in the $\mathrm{FC}$ test consisted of seven positive and seven negative faces $(\mathrm{P} \%=0,10,20,35,50$, 75 , and 100), including the positive and negative training stimuli $(\mathrm{P} \%=35)$ and their "parents" $(\mathrm{P} \%=0$ and 100$)$. 
For example, the pigeon trained to discriminate the AM face from the EN face was then tested with the seven faces on the top face-morph dimension shown in Fig. 2 and the corresponding series of seven faces from the other stimulus set. Each stimulus was displayed once in each of four blocks of 14 trials. In the NC test, each pigeon received generalization testing with a series of seven faces on one of the two lower facemorph dimensions shown in Fig. 2, as well as a series of seven faces from the other stimulus set. The stimuli from the two different facemorph dimensions in each stimulus set were tested equally often among 4 pigeons. Other procedural details were the same as those in Experiment 1.

\section{Results}

The pigeons took a mean of 5.8 (range: 4-9) days to reach the $80 \%$ criterion, but they all received 12 training sessions. The mean numbers of responses in the subsequent $\mathrm{FC}$ and NC tests were 562.5 (range: 446-728) and 816.3 (range: 623-982), respectively. The pigeons responded considerably more in the NC test, which was mainly due to the higher rates of responses to the negative stimuli in this test.

The upper left panel in Fig. 4 shows the mean generalization gradient in the $\mathrm{FC}$ test. It peaked at the positive training stimulus, with no or few responses to all the negative stimuli. As can be seen in the lower left panel, however, there was a substantial individual variability in shape of generalization gradients. The stimulus pairs differing among the pigeons were used for the single positive and single negative training that did not necessarily require them to discriminate the stimuli (e.g., the AM and $\mathrm{EN}$ faces) by relying on the $\mathrm{M}$ and $\mathrm{N}$ components. This might have resulted in a good deal of variability. The right panels in Fig. 4 show the results from the NC test. The pigeons showed similar overall patterns; the generalization gradient increased as a function of the $\mathrm{P}+$ proportion and decreased as a function of the P-proportion. This was so in bird 1 , but only this bird showed relatively higher response rates to the negative stimuli than to the positive ones. Perhaps, the novel idiosyncratic component(s) of the negative category were accidentally similar to this bird's positive training stimulus.

A two-way repeated measures ANOVA was conducted on the data for the positive test stimuli, with test (FC vs. NC) and morph proportion ( $0 \%$ vs. $10 \%$ vs. $20 \%$ vs. $35 \%$ vs. $50 \%$ vs. $75 \%$ vs. $100 \%$ ) as within-subjects variables. Because there was a significant interaction between these two factors, $F(6$, $18)=4.31, p=.007$, we performed a simple main effects analysis of morph proportion. The effect, $\mathrm{F}(6,36)=1.28$, $p=.292$, was not significant in the FC test. In contrast, the effect, $\mathrm{F}(6,36)=4.05, p=.003$, was significant in the $\mathrm{NC}$ test.
A one-way repeated measures ANOVA with morph proportion as a within-subjects variable was performed on the data for the negative stimuli in the $\mathrm{NC}$ test (the data from the FC test were excluded, because they showed no variation, so could only have reduced the power of the test). The effect was not significant, $\mathrm{F}(6,18)=1.95, p=.28$.

\section{Discussion}

The generalization gradient in the FC test revealed that the pigeons discriminated the faces on the positive and negative face-morph dimensions in virtually an all-or-none fashion, although there was a considerable variation in shape of the positive generalization gradients. This finding, in turn, suggests that categorization training in Experiment 1 increased distinctiveness of the exemplars within categories, to the extent that the pigeons responded to the test stimuli in a "graded" fashion according to the $\mathrm{P}+$ and $\mathrm{P}$-proportions. Such an effect has been known as acquired distinctiveness; there is an increase in perceptual sensitivity to differences that are relevant for a categorization (Corneille \& Judd, 1999; Gibson, 1969; Goldstone, 1994; see also Aitken, Bennett, McLaren, \& Mackintosh, 1996).

An additional important finding in the $\mathrm{FC}$ test is that the A, B, C, or D face was well discriminated from the E, F, G, or $\mathrm{H}$ face $(\mathrm{P} \%=0)$. This finding, in turn, suggests that categorization training acted to decrease distinctiveness of the idiosyncratic component faces in the different categories in Experiment 1. This might be even more so for those belonging to the same category, as we will discuss later.

In the NC test, the pigeons' rate of responding increased as a function of the $\mathrm{P}+$ proportion and decreased as a function of the P-proportion. This result can be explained by supposing that the pigeons relied on a familiarity/ novelty judgment. An earlier study by Cerella (1979) demonstrated that pigeons trained with just one exemplar of a natural category, oak leaf, then successfully classified novel pictures of a variety of oak leaves, discriminating them from pictures of other species of leaves. Undoubtedly, such an economy in classifying novel stimuli on the basis of a familiarity/novelty judgment can happen because of the structure of basic-level categories.

However, it is premature to conclude that the pigeons in Experiment 1 also responded on the basis of a familiarity/ novelty judgment in the NIC test. In Experiment 2, the mean response rates (responses/minute) to $\mathrm{P}+$ were 19.7 (range: 6.8-29.3) in the FC test and 71.7 (range: 27.0128.3) in the NC test. The magnitude of the response strength to $\mathrm{P}+$ depended on the test stimulus sets - an effect similar to the response range effect; the location of the peak of responding depends on the range of stimuli used in testing. Response range effects have been found in humans (Thomas, 1993, 1994) and explained by Thomas (1993) on 

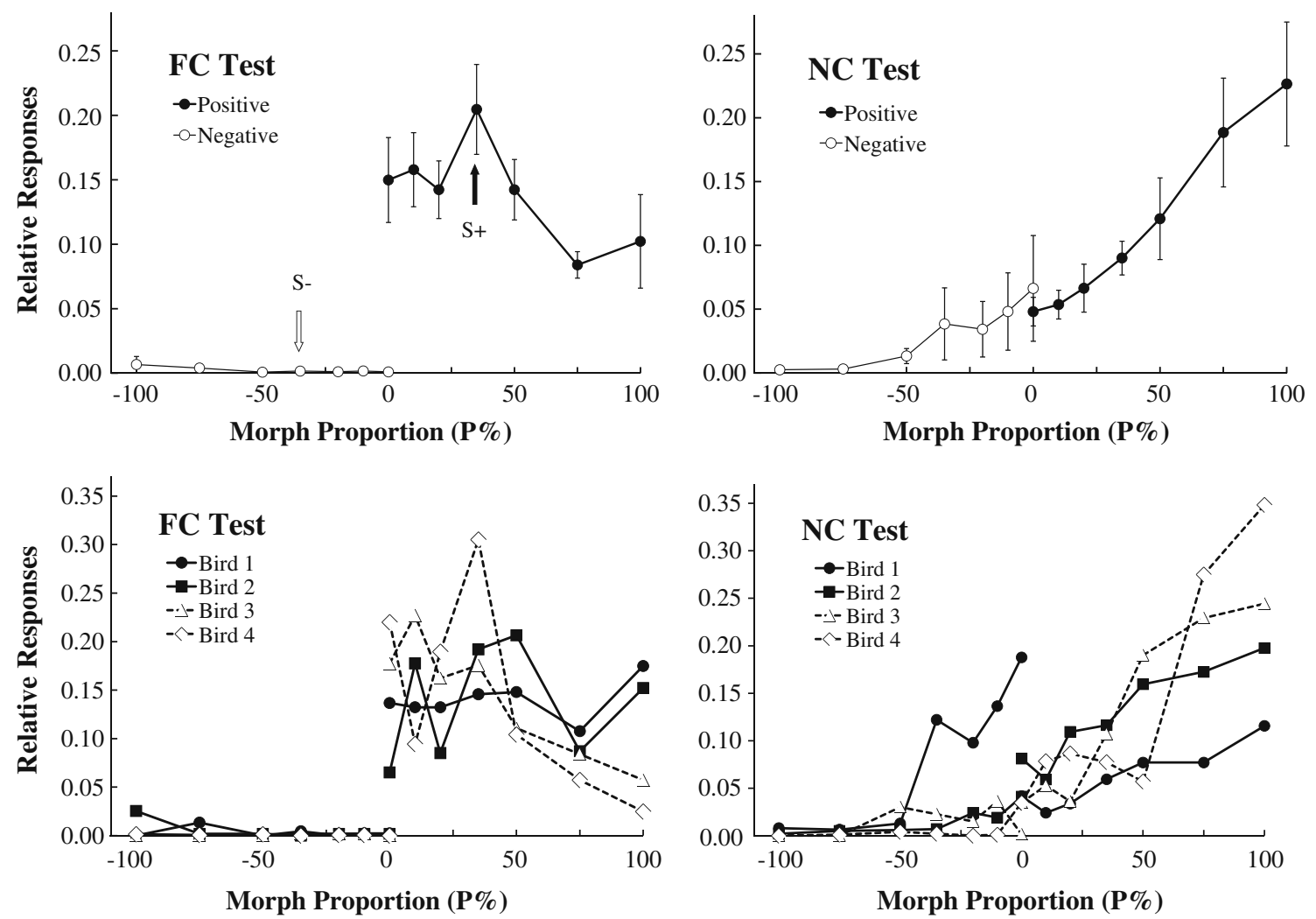

Fig. 4 The upper panels show mean relative responses to the positive and negative test stimuli as a function of the proportion of the common component in the familiar component (FC) test and the novel component (NC) test in Experiment 2. The error bars show standard

errors. The white and black arrows indicate which datapoints were observed for the negative ( $\mathrm{S}-)$ and positive $(\mathrm{S}+)$ stimuli, respectively. The lower panels show a generalization gradient for each pigeon

the basis of Helson's (1964) adaptation-level theory. Given that the test stimuli appearing during the FC test are more physically similar to the training exemplars than are those appearing during the $\mathrm{NC}$ test, the present finding is consistent with the notion applied to the response range effects. As we described earlier, the novel negative idiosyncratic components might have been very similar to the familiar positive stimulus used for bird 1. Interestingly, only this bird did not show such an effect.In Experiment 1, the mean response rates to $\mathrm{P}+$ in the FIC and NIC tests were 63.3 (range: 48.0-77.3) and 65.6 (range: 40.5-84.8) for Group $35+35$ - and 62.2 (range: $39.0-82.5$ ) and 60.3 (range: 39.4-70.9) for Group $35+50-$, respectively. Thus, the effect similar to the response range effect was not found. The two stimulus sets might be perceptually similar for the pigeons in Experiment 1 (but not for the pigeons in Experiment 2, including bird 1), and they behaved in the NIC test virtually in the same way as in the FIC test. Excellent transfer to the novel stimuli shown by the pigeons in Experiment 1 is in accord with the principle of acquired equivalence; there is a decrease in perceptual sensitivity to differences that are not relevant for a categorization (Goldstone, 1994; Pearce \& Hall, 1980).

Acquired equivalence and distinctiveness, as we have discussed in the context of perceptual learning (e.g., Goldstone, 1998), are not processes that conflict with one another but consistently indicate that the pigeons in Experiment 1 were focusing on, or attending to, the common component features (i.e., the general, diagnostic aspects of the categories) to classify the stimuli. This might have produced the generalization gradient in the NIC test comparable to that in the FIC test. It seems that generalization from the learned features that are specific to the composite faces used for training is insufficient to explain the pigeons' flexible classification performance.

\section{Experiment 3}

None of the idiosyncratic components for the categories used in Experiment 1 are overlapping between the categories. This may not necessarily be the case for the categories in the real world. Among the idiosyncratic components for the categories used in this experiment, some are overlapping between the categories and others are not. The positive and negative stimuli containing the same 
idiosyncratic component are substantially similar to one another and can be discriminated only with the common component features. One may predict that pigeons would have difficulty in discriminating these stimuli but eventually would learn to classify all the stimuli on the basis of the common component features. Then, generalization gradients for these stimuli, as well as the stimuli containing the nonoverlapping idiosyncratic components, will not largely differ from those obtained in Experiment 1. An opposite prediction is as follows. For the stimuli containing an overlapping idiosyncratic component (e.g., XP+ and XP-), the $\mathrm{P}+$ and $\mathrm{P}-$ components are both associated with a third component ( $\mathrm{X}$ in this example). This would make it harder to discriminate the stimuli relying on the $\mathrm{P}+$ and $\mathrm{P}$ - component features (see Honey \& Hall, 1989, 1991), and pigeons will make use of the nonoverlapping idiosyncratic component features when they are available.

Hereafter, the idiosyncratic components that are not overlapping between the positive and negative categories as in Experiment 1 will be referred to as $\alpha+$ and $\alpha-, \beta+$ and $\beta$-, and $\gamma+$ and $\gamma$-, to distinguish these components from those that are overlapping between the categories (X, Y, and Z).

\section{Method}

Animals, stimulus materials, and apparatus Four new pigeons that had no prior experience with pictorial stimuli served as subjects. Nine faces were selected from those used as the familiar and novel idiosyncratic components in Experiment 1 . Six of these faces $(\alpha+, \beta+$, and $\gamma+$ in the positive category; $\alpha-, \beta$-, and $\gamma$ - in the negative category) were not overlapping between the categories, while the remaining three faces $(\mathrm{X}, \mathrm{Y}$, and $\mathrm{Z})$ were overlapping. In each category, $\mathrm{P}$ was morphed into $\alpha, \beta$, and $\gamma$; into their respective $50 \%$ morphs $\alpha \beta, \alpha \gamma$, and $\beta \gamma$; into $\mathrm{X}, \mathrm{Y}$, and $\mathrm{Z}$; and into their respective $50 \%$ morphs $\mathrm{XY}, \mathrm{XZ}$, and $\mathrm{YZ}$. A total of 12 training stimuli in each category contained $35 \% \mathrm{P}$. The apparatus was the same as in the previous experiments, except that four identical chambers were used (one for each pigeon).

Procedure A training session consisted of three blocks of 24 trials, with each stimulus presented once in a block. Each pigeon received a minimum of 14 sessions, and they continued until the $80 \%$ criterion was attained for each of the nonoverlapping and overlapping idiosyncratic component stimulus sets in each of two consecutive daily sessions. The pigeons then received generalization testing on the positive and negative face-morph dimensions ranging from $\alpha, \beta, \gamma, \mathrm{X}, \mathrm{Y}$, or $\mathrm{Z}$ to $\mathrm{P}$. Table 1 describes the structure of the stimulus set used for generalization testing. Each of the 30 positive and 30 negative morphs $(\mathrm{P} \%=10,20,35,50$, and 75 on the six face-morph dimensions), as well as $\alpha+$,
Table 1 The stimulus set used for generalization testing in Experiment 3

\begin{tabular}{lllllll}
\hline \multicolumn{7}{l}{ Morph Proportion $(P \%)$} \\
\hline $0 \%$ & $10 \%$ & $20 \%$ & $35 \%$ & $50 \%$ & $75 \%$ & $100 \%$ \\
\hline$\alpha+$ & $\alpha \mathrm{P}+$ & $\alpha \mathrm{P}+$ & $\alpha \mathrm{P}+$ & $\alpha \mathrm{P}+$ & $\alpha \mathrm{P}+$ & $\mathrm{P}+$ \\
$\beta+$ & $\beta \mathrm{P}+$ & $\beta \mathrm{P}+$ & $\beta \mathrm{P}+$ & $\beta \mathrm{P}+$ & $\beta \mathrm{P}+$ & $\mathrm{P}+$ \\
$\gamma+$ & $\gamma \mathrm{P}+$ & $\gamma \mathrm{P}+$ & $\gamma \mathrm{P}+$ & $\gamma \mathrm{P}+$ & $\gamma \mathrm{P}+$ & $\mathrm{P}+$ \\
$\mathrm{X}$ & $\mathrm{XP}+$ & $\mathrm{XP}+$ & $\mathrm{XP}+$ & $\mathrm{XP}+$ & $\mathrm{XP}+$ & $(\mathrm{P}+)$ \\
$\mathrm{Y}$ & $\mathrm{YP}+$ & $\mathrm{YP}+$ & $\mathrm{YP}+$ & $\mathrm{YP}+$ & $\mathrm{YP}+$ & $(\mathrm{P}+)$ \\
$\mathrm{Z}$ & $\mathrm{ZP}+$ & $\mathrm{ZP}+$ & $\mathrm{ZP}+$ & $\mathrm{ZP}+$ & $\mathrm{ZP}+$ & $(\mathrm{P}+)$ \\
$\alpha-$ & $\alpha \mathrm{P}-$ & $\alpha \mathrm{P}-$ & $\alpha \mathrm{P}-$ & $\alpha \mathrm{P}-$ & $\alpha \mathrm{P}-$ & $\mathrm{P}-$ \\
$\beta-$ & $\beta \mathrm{P}-$ & $\beta \mathrm{P}-$ & $\beta \mathrm{P}-$ & $\beta \mathrm{P}-$ & $\beta \mathrm{P}-$ & $\mathrm{P}-$ \\
$\gamma-$ & $\gamma \mathrm{P}-$ & $\gamma \mathrm{P}-$ & $\gamma \mathrm{P}-$ & $\gamma \mathrm{P}-$ & $\gamma \mathrm{P}-$ & $\mathrm{P}-$ \\
$(\mathrm{X})$ & $\mathrm{XP}-$ & $\mathrm{XP}-$ & $\mathrm{XP}-$ & $\mathrm{XP}-$ & $\mathrm{XP}-$ & $(\mathrm{P}-)$ \\
$(\mathrm{Y})$ & $\mathrm{YP}-$ & $\mathrm{YP}-$ & $\mathrm{YP}-$ & $\mathrm{YP}-$ & $\mathrm{YP}-$ & $(\mathrm{P}-)$ \\
$(\mathrm{Z})$ & $\mathrm{ZP}-$ & $\mathrm{ZP}-$ & $\mathrm{ZP}-$ & $\mathrm{ZP}-$ & $\mathrm{ZP}-$ & $(\mathrm{P}-)$ \\
\hline
\end{tabular}

Note: The stimuli in parentheses are not used. There are a total of 75 stimuli

$\beta+, \gamma^{+}, \alpha-, \beta-, \gamma-, X, Y$, and $\mathrm{Z}$ appeared once in a session, while $\mathrm{P}+$ and $\mathrm{P}$ - appeared three times in a session. Three blocks of 25 trials were prepared, so that the different idiosyncratic components, as well as the different morph proportions, were tested as equally often as possible in each block. Four sessions were given. Sequences of the preset blocks were randomized among sessions and pigeons. The total display time for the stimuli with a given morph proportion was $240 \mathrm{~s}$ over four sessions ( $60 \mathrm{~s}$ in each), with each individual stimulus presented a total of $80 \mathrm{~s}$ during the test. Retraining intervened as in Experiment 1.

\section{Results and discussion}

Training The average number of sessions required to reach $80 \%$ or better accuracy for two consecutive days was 8.0 (range: 5-12) and 9.0 (range: 4-16) for the stimuli containing the nonoverlapping and overlapping idiosyncratic components, respectively. A significant difference was not found in the number of required sessions, $\mathrm{F}(1,3)=3.08, p=.129$. Further analyses will be done after Experiment 4, in which 4 additional pigeons received training in exactly the same way as in the present experiment.

Generalization testing Figure 5 shows mean relative responses plotted as a function of the $\mathrm{P}$ proportion. The overlapping idiosyncratic components resulted in a steeper positive generalization gradient than did the nonoverlapping ones. A similar but less apparent tendency was observed with the negative generalization gradients. A two-way repeated measures ANOVA with idiosyncratic 


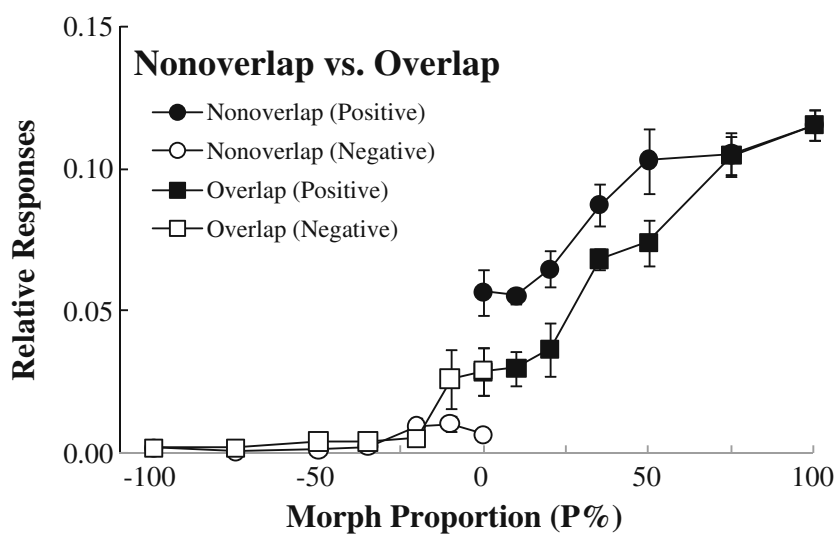

Fig. 5 Mean relative responses to the positive and negative test stimuli as a function of the proportion of the common component in Experiment 3. The idiosyncratic components are overlapping or not overlapping between the categories. The error bars show standard errors

component (nonoverlapping vs. overlapping) and morph proportion ( $0 \%$ vs. $10 \%$ vs. $20 \%$ vs. $35 \%$ vs. $50 \%$ vs. $75 \%$ ) as within-subjects variables was separately conducted on the data for the positive and negative stimuli. For the positive stimuli, the main effects $[\mathrm{F}(1,3)=16.69, p=.026$, for idiosyncratic component; $\mathrm{F}(5,15)=16.28, p<.001$, for morph proportion] were both significant. The interaction, $\mathrm{F}$ $(5,15)=1.95, p=.146$, was not significant. For the negative stimuli, only the effect of morph proportion, $\mathrm{F}(5$, $15)=9.66, p<.001$, was significant.

The pattern of results suggests that although the pigeons attended more to the common component features and less to the idiosyncratic component features, they also made use of the nonoverlapping idiosyncratic features to classify the stimuli. However, one may argue that the pigeons were more sensitized to the difference in $\mathrm{P}$ proportion of the stimuli that had the overlapping idiosyncratic components but did not make use of the nonoverlapping idiosyncratic features when the common component features were available $(\mathrm{P} \%>0)$.

\section{Experiment 4}

This experiment trained new pigeons in exactly the same way as in Experiment 3 and then tested whether they learned to make use of the nonoverlapping idiosyncratic features, by reversing the components $\alpha, \beta$, and $\gamma$ between the positive and negative categories during generalization testing.

\section{Method}

Animals, stimulus materials, and apparatus Four new pigeons that had no prior experience with pictorial stimuli served as subjects. Stimulus materials and apparatus were the same as those in Experiment 3, except for the additional test stimuli that contained the idiosyncratic components of the opposite category $(\alpha-\mathrm{P}+, \beta-\mathrm{P}+$, and $\gamma-\mathrm{P}+$ in the positive category; $\alpha+\mathrm{P}_{-}, \beta+\mathrm{P}-$, and $\gamma+\mathrm{P}-$ in the negative category).

Procedure The pigeons were trained to discriminate the categories in the same way as in Experiment 3. Two pigeons then received generalization testing as in Experiment 3, except that the nonoverlapping components $\alpha, \beta$, and $\gamma$ were reversed between the categories (reversed vs. overlap component test). The remaining 2 pigeons received generalization testing with the composite faces, all of which contained nonoverlapping idiosyncratic components, but these components were reversed or not reversed during testing (reversed vs. nonreversed component test). For these 2 pigeons, a test session consisted of three blocks of 24 trials. Each of the 30 positive and 30 negative morphs $(\mathrm{P} \%=10$, $20,35,50$, and 75 on the six facemorph dimensions), as well as $\alpha+, \beta+, \gamma^{+}, \alpha-, \beta-$, and $\gamma^{-}$, appeared once in a session, while $\mathrm{P}+$ and $\mathrm{P}-$ appeared three times in a session. Other procedural details were the same as those in Experiment 3.

\section{Results and discussion}

Training The number of sessions required to reach $80 \%$ or better accuracy for two consecutive days averaged 7.0 (range: 5-9) and 10.25 (range: 5-16) for the stimuli containing the nonoverlapping and overlapping idiosyncratic components, respectively. A significant difference in the number of required sessions was not found, $\mathrm{F}(1,3)=$ $4.74, p=.118$.

All the pigeons received a minimum of 14 training sessions, as in Experiment 3. For the pigeons in Experiments 3 and 4, performances to the stimuli containing the nonoverlapping and overlapping idiosyncratic components were compared at different acquisition stages. Figure 6 shows the mean DRs in the first sessions where each pigeon reached $60 \%$ or better

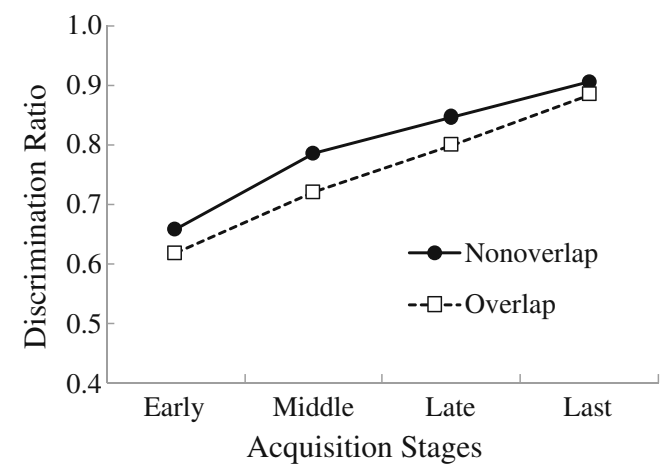

Fig. 6 Mean discrimination ratios at different acquisition stages in Experiments 3 and 4 
overall accuracy (early), $70 \%$ or better overall accuracy (middle), or $80 \%$ or better overall accuracy (late) and in the last training session (last). Over the course of classification training, performance to the stimuli containing the nonoverlapping idiosyncratic components was slightly, but consistently, better than that to the stimuli containing the overlapping ones. A two-way repeated measures ANOVA with stimulus and acquisition stage as within-subjects variables revealed that the main effects $[\mathrm{F}(1,7)=6.10$, $p=.043$, for stimulus; $\mathrm{F}(3,21)=64.92, p<.001$, for acquisition stage] were both significant. The interaction was not significant, $\mathrm{F}(3,21)=0.41, p=.747$. These findings will be discussed in the General Discussion section.

Generalization testing Figure 7 shows mean relative responses plotted as a function of the $\mathrm{P}$ proportion. The reversed idiosyncratic components resulted in a much lower classification accuracy than did the nonreversed ones (the upper panel) and also less classification accuracy than did the overlapping ones (the lower panel). A two-way repeated
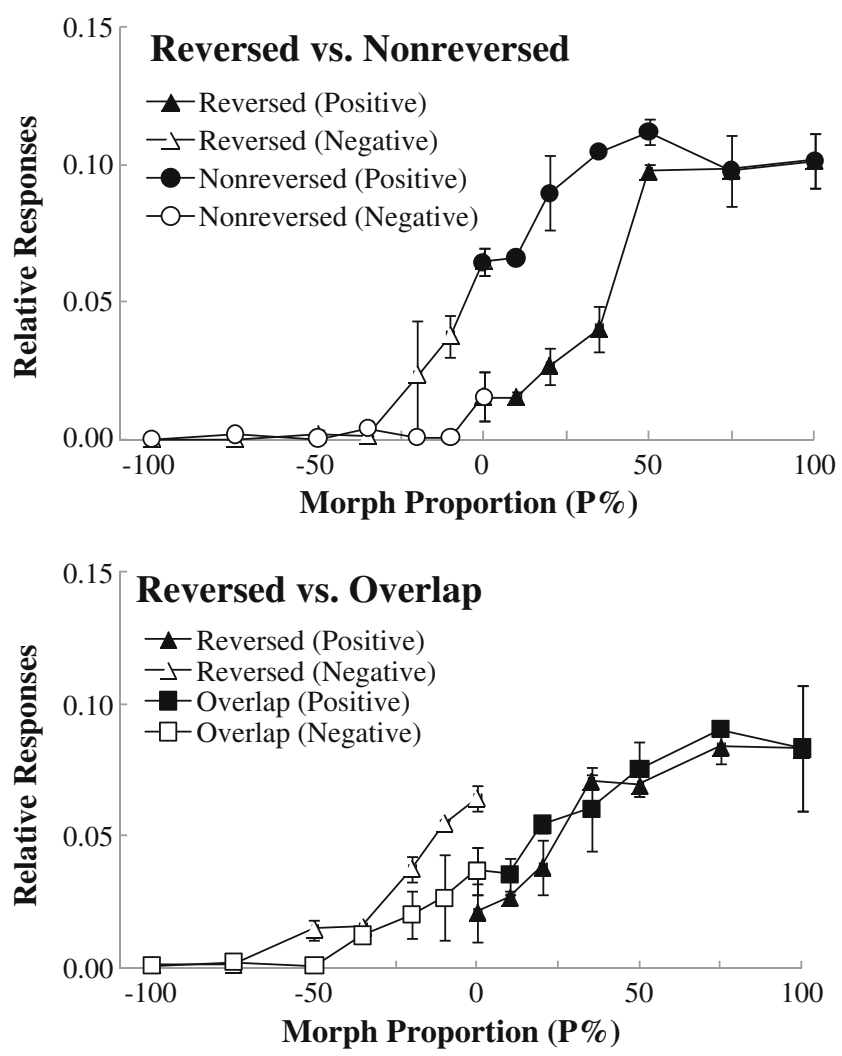

Fig. 7 Mean relative responses to the positive and negative test stimuli as a function of the proportion of the common component in Experiment 4. In the "reversed vs. nonreversed" test, the nonoverlapping components are reversed or not reversed between the categories. In the "reversed vs. overlap" test, the idiosyncratic components are overlapping or not overlapping between the categories, but the nonoverlapping ones are reversed between the categories. The error bars show standard errors measures ANOVA with idiosyncratic component (reversed vs. nonreversed) and morph proportion ( $0 \%$ vs. $10 \%$ vs. $20 \%$ vs. $35 \%$ vs. $50 \%$ vs. $75 \%$ ) as within-subjects variables was conducted on the data shown in the upper panel. For the positive stimuli, the main effects $[\mathrm{F}(1,1)=3735.00$, $p=.01$, for the idiosyncratic component; $\mathrm{F}(5,5)=18.56$, $p=.003$, for morph proportion $]$ and the interaction $[\mathrm{F}(5,5)=$ $10.28, p=.012]$ were significant. A simple main effects analysis of the idiosyncratic component revealed the significant differences for $\mathrm{P} \%=0[\mathrm{~F}(1,6)=41.24, p<.001]$, $\mathrm{P} \%=10[\mathrm{~F}(1,6)=44.18, p<.001], \mathrm{P} \%=20[\mathrm{~F}(1,6)=$ $67.34, p<.001]$, and $\mathrm{P} \%=35[\mathrm{~F}(1,6)=71.43, p<.001]$. For the negative stimuli, the effect of morph proportion, $\mathrm{F}(5,5)=9.73, p=.013$, and the interaction, $\mathrm{F}(5,5)=7.06$, $p=.026$,were significant. A simple main effects analysis of the idiosyncratic component revealed the significant differences at $\mathrm{P} \%=0[\mathrm{~F}(1,6)=33.2, p=.001], \mathrm{P} \%=10[\mathrm{~F}(1,6)=$ $18.42, p=.005]$, and $\mathrm{P} \%=20[\mathrm{~F}(1,6)=6.9, p=.039]$.

A two-way repeated measures ANOVA with the idiosyncratic component (reversed vs. overlap) and morph proportion ( $0 \%$ vs. $10 \%$ vs. $20 \%$ vs. $35 \%$ vs. $50 \%$ vs. $75 \%$ ) as within-subjects variables was conducted on the data shown in the lower panel. For the positive stimuli, only the main effects $[\mathrm{F}(1,1)=262.20, p=.039$, for idiosyncratic component; $\mathrm{F}(5,5)=9.87, p=.013$, for morph proportion] were significant. For the negative stimuli, only the effect of morph proportion was significant, $\mathrm{F}(5,5)=10.65, p=.011$.

The composite faces containing the same proportion of reversed and nonreversed idiosyncratic components are, by definition, the same distance away from the prototype in a multidimensional stimulus space. It is impossible to distinguish these faces without knowing which idiosyncratic feature belongs to which category. Nevertheless, the pigeons appeared to distinguish these stimuli when the stimuli had relatively small proportions of the common component. Even when the proportion decreased to $0 \%$, the mean DR was .82 (it was .89 in Experiment 3). An important implication of this result is that the nonoverlapping idiosyncratic features enabled the pigeons to classify the various exemplars that had no or quite a few features in common. A question left open is whether the pigeons have learned functional equivalence for the nonoverlapping idiosyncratic features that are associated with the general aspect of the category, as well as a common consequence during categorization training (see von Fersen \& Lea, 1990, for an attempt to explore feature equivalence in pigeons).

Our conclusion is that, although the pigeons attended more to the common component features and less to the idiosyncratic component features, they also made use of the idiosyncratic features that were not overlapping between the categories. They were more inclined to do so than the pigeons in Experiment 1 were. This finding will be more fully discussed in the General Discussion section. 


\section{General discussion}

The present study investigated categorization by pigeons, using artificial categories constructed by mimicking the structure of basic-level categories. We used a morphing technique that allowed us to investigate the contributions of the common and idiosyncratic components of exemplars, although we cannot identify physical features actually used by pigeons to discriminate the multifaceted stimuli. The prototype effect robustly shown by the 16 pigeons (Experiments 1, 3 and 4) suggested that they classified the various exemplars in the manner that maximized similarity within the categories and dissimilarity between the categories.

\section{Classification models}

Feature learning As we discussed in Experiments 1 and 2, the feature-learning-and-generalization account is insufficient as a basis for the pigeons' flexible classification performance with the stimuli containing novel idiosyncratic components. It should be noted here that the feature-based account predicts that features that are similar across all or most members control classification performance early in training, whereas features that are specific to individual members are learned later in training (see also Soto \& Wasserman, 2010, for a similar prediction from their common elements model). Then, the advantage of the stimuli containing the nonoverlapping idiosyncratic components to the stimuli containing the overlapping ones should grow larger at later stages of training in Experiments 3 and 4. This is not in agreement with the pattern of results shown in Fig. 6. All in all, the feature-based account proposed in Experiment 1 may not be suited to explain the prototype effect shown by the pigeons.

Prototype models Did the pigeons learn the categories represented by the unseen positive and negative prototypes and then respond to the test stimuli relying on the degree to which each stimulus represents the abstracted categories?

In the present study, the prototype that, in fact, resulted in the pigeons' best classification performance is defined independently of idiosyncratic features. The stimuli that have the same proportion of the overlapping, nonoverlapping, or reversed idiosyncratic components are, by definition, the same distance away from the prototypes. Perhaps an advantage of the prototype-based strategy is that, once the prototype has been learned, it enables subjects to classify the stimuli, regardless of the idiosyncratic component features. However, the pigeons did not demonstrate the advantage in the generalization tests in Experiments 3 and 4. Distancefrom-prototype models (Posner \& Keele, 1968; Reed, 1972; Smith \& Minda, 1998) are not tenable to explain the findings in Experiments 3 and 4. Furthermore, the pigeons in these experiments accurately discriminated the positive and negative idiosyncratic component faces $(\mathrm{P} \%=0)$, a finding indicating that they made use of the nonoverlapping idiosyncratic features to classify the stimuli. It is still possible to assume the use of a prototype-based strategy for the pigeons in Experiment 1, but we believe that classification strategies adopted by the pigeons may not differ so drastically between the experiments.

Exemplar memorization One may argue that the pigeons first learn and abstract the prototype and then learn the individual exemplars (e.g., a mixed model of Smith \& Minda, 2000). The prototype + exemplar memorization model predicts that (1) the generalization gradient has a second peak at the training exemplars, or otherwise the gradient becomes shallow over the range between the training exemplars and the prototype;(2) performance to the training exemplars in the FIC test is greater than that to the corresponding novel stimuli in the NIC test; and (3) the advantage of the stimuli containing the nonoverlapping idiosyncratic components should grow at a later stage of training. Our results are contrary to these predictions.

It has been reported that nonhumans have difficulty in learning XOR (exclusive-or) categories (e.g., Smith, Coutinho, \& Couchman, 2010). A simplest XOR category consists of the exemplars (11 and 00$)$, and the other category of the exemplars (10 and 01). For example, yellow squares and blue circles are the members of one category, and yellow circles and blue squares are the members of the other category. The categories are not linearly separable from one another, requiring animals to learn to respond or not to respond to each individual stimulus or to discover, if possible at all, a rule-based solution. Learning of the XOR category would be very difficult, simply because animals have to respond similarly to completely dissimilar stimuli and differently to substantially similar stimuli. In the present study, by contrast, the pigeons learned the categories with ease and had no difficulty in classifying novel exemplars of the categories. The pigeons' excellent classification performance confirms that the various exemplars have formed well-structured, linearly separable categories. One possible solution to such a classification task, which may be cognitively more economic than learning separate individual stimuli, is a contextdependent exemplar-based categorization.

Exemplar-based categorization An exemplar model well known as the generalized context model(GCM) is able to predict diverse patterns of classification performance by selectively tuning adjustable attentional weights on multidimensional stimulus dimensions (Nosofsky, 1986, 1991). Nosofsky described attention shifts in terms of psychological "stretching" of relevant dimensions and "shrinking" of 
irrelevant dimensions. GCM assumes that classification decisions are based on the similarity of an item to the exemplars of a target category, relative to the exemplars of nontarget categories - a decision rule referred to as the relative-similarity measure. Nosofsky (1991) argued, however, that an important determinant of typicality judgments often involves the absolute summed similarity of an item to the exemplars of the target category. Perhaps, for the stimuli that contained the nonoverlapping idiosyncratic components, similarity to the exemplars of the opposite category was too small to contribute to the typicality judgments, so that the absolute-similarity measure is suited to predict our findings with these stimuli. It predicts that the generalization gradients will increase as a function of the $\mathrm{P}+$ proportion and decrease as a function of the $\mathrm{P}$ - proportion by selecting appropriate attentional weights for the common and idiosyncratic component features. In a situation in which subjects attend more to the common component features and less to the idiosyncratic component features, similarity between the common component face and each exemplar of its category (e.g., ${ }_{\mathrm{M}} \mathrm{S}_{\mathrm{AM}}$ in category 1) will be larger than similarity between two exemplars (e.g., $\mathrm{BM}_{\mathrm{AM}}$ ), and much larger than similarity between an idiosyncratic component face and the exemplar containing that component (e.g., ${ }_{\mathrm{A}} \mathrm{S}_{\mathrm{AM}}$ ). Thus, ${ }_{\mathrm{A}} \mathrm{S}_{\mathrm{AM}}<{ }_{\mathrm{BM}} \mathrm{S}_{\mathrm{AM}}<{ }_{\mathrm{M}} \mathrm{S}_{\mathrm{AM}}$. Balance between the attentional weights allows one to predict the different slopes of generalization gradients observed in the present experiments.

By contrast, for the stimuli that contained the overlapping idiosyncratic components, similarity to the exemplars of the opposite category may have contributed to the typicality judgments, so that the relative-similarity measure is suited to predict our findings with these stimuli. The relative-similarity measure predicts a generalization gradient increasing as a function of the $\mathrm{P}+$ proportion and decreasing as a function of the $\mathrm{P}$ - proportion on a hypothetical similarity dimension ranging from $\mathrm{P}$ - to the idiosyncratic component faces and to $\mathrm{P}+$, when similarity is assumed to be based on a Gaussian function of stimulus distance.

GCM is not acting as a rote memorizer that has lost any sense of category structure (Nosofsky, 2000); it assumes that subjects distribute attention among the stimulus dimensions so as to optimize performance in each given categorization problem. Our findings favor the exemplarbased categorization. However, because GCM is a static model intended to account for categorization under fairly stable performance conditions, it does not allow us to explain the dynamic process by which the attentional weights are adjusted.

\section{Attention shift}

An exemplar-based connectionist model called the attention learning covering map(ALCOVE) extends GCM by adding a mechanism of attention learning (Kruschke, 1992). It applies only to situations in which the stimuli can be appropriately represented as points in a multidimensional stimulus space. In the present study, it was especially hard to prepare the hypothetical stimulus sets for Experiments 3 and 4 that would have made our simulations manageable while maintaining the essence of morphed face stimuli sufficiently realistic. ${ }^{1}$

Although we do not propose an alternative, quantitative, model that may predict our findings, it may be worth noting the possibility of qualitative prediction of the attention shift shown by the pigeons. We assume that the magnitude of response to a given exemplar is determined by the sum of associative strength of the common and idiosyncratic components of the exemplar. For example, the magnitude of response to the AM face is determined by the sum of associative strength of the common component $\mathrm{M}$ and its idiosyncratic component A. The findings in Experiment 1 suggest that the common component acquired most of the associative strength to the outcome. When exemplars containing the idiosyncratic components that are also shared with exemplars of the opposite category (i.e., the $\mathrm{XM}$, YM, and ZM faces in category 1 and the XN, YN, and $\mathrm{ZN}$ faces in category 2) are introduced, the inclusiveness of the categories will change so as to correctly classify the somewhat similar positive and negative exemplars. The common component shared even with these poor exemplars of the category would make it difficult to acquire the associative strength to the outcome, leaving more strength to be acquired for the nonoverlapping idiosyncratic components (the A, B, C, and D components in category 1 and the E, F, G, and $\mathrm{H}$ components in category 2). On the other hand, the associative strength of the idiosyncratic components overlapping between the positive and negative

\footnotetext{
${ }^{1}$ A possible way to simulate Experiment 1 is to suppose a threedimensional stimulus space, in which the idiosyncratic components that were arbitrarily assigned to the two categories lie on the same surface, while the common components lie on other different surfaces. Preliminary simulations carried out by our colleague, Toshihiko Matsuka, showed that the ALCOVE exhibited the prototype effect, but with a very small free parameter that controlled the overall similarity gradient in the stimulus space. In other words, the ALCOVE had to be insensitive to the individual exemplars (i.e., each exemplar had a very broad receptive coverage area and created one big activation area per category, like prototype models). This finding seems to be consistent with our finding that the pigeons in Experiment 1 in fact treated the idiosyncratic components in each of the two distinctive categories more or less equivalently. However, for simulations of Experiments 3 and 4, we are currently unable to include the hypothetical stimuli containing overlapping idiosyncratic components into the stimulus space used for the simulations of Experiment 1; the stimuli should be more similar across the categories than are the stimuli containing the nonoverlapping idiosyncratic components, while these different types of stimuli should be the same distance away from the prototype within the categories.
} 
exemplars (the $\mathrm{X}, \mathrm{Y}$, and $\mathrm{Z}$ components) would remain at zero, and, therefore, classification performance with the poor exemplars containing these idiosyncratic components might be degraded to some extent over the course of acquisition. Perhaps, to reach an asymptotic level of associative learning, if necessary, for the exemplars containing the overlapping idiosyncratic components, exemplar-based memorization is required at a later stage of acquisition (see also Cook \& Smith, 2006, for exemplar-based memorization of exception items that do not fit categories' similarity and dissimilarity structure).

Attention shift requires a model that allows us to represent the same stimulus in different ways depending on the context in which it is presented (see also Harris, 2006). Although our approach needs a greater refinement, it successfully illustrates the course of category learning depicted in Fig. 6, as well as the process by which the attentional weights are adjusted so as to optimize classification performance during training. Perhaps, the basic learning mechanism could be retained even with exemplars consisting of separable, easily identifiable features, if they were perceived in a configural manner and, hence,were represented by the general and idiosyncratic aspects of the category.

\section{Conclusions}

Exemplar-based categorization is sufficient to explain the prototype effect shown by the pigeons in the present study, without a need to posit a prototype abstraction process. Categorization accomplished by attending more to general, diagnostic aspects and less to idiosyncratic aspects gives rise to acquired distinctiveness and equivalence, which optimizes similarity relations for the categories. Such a prelinguistic competence could have presumably evolved in most animals, including humans, as a result of the demand that their cognitive systems experience-namely, to reduce the limitless variation in the world to manageable, coherent object classes. This built-in mechanism could strongly contribute to the classification of concrete objects by humans and nonhuman animals in their living environments. Additional studies will shed further light on the nature of the processes operating in the categorization of natural objects.

Acknowledgments This research was supported by a research grant from the Japan Society for Promotion of Science to M.J. We thank Yuriko Tanaka, Masumi Sugiyama, Kaori Watanabe, and Maiko Naito for their assistance with some of the phases of the present study, and Toshihiko Matsuka for fruitful discussions. We are grateful to Nocholas Mackintosh for his suggestions, which influenced this work, and anonymous reviewers for their thoughtful comments.

\section{References}

Aitken, M. R. F., Bennett, C. H., McLaren, I. P. L., \& Mackintosh, N. J. (1996). Perceptual differentiation during categorization learning by pigeons. Journal of Experimental Psychology: Animal Behavior Processes, 22, 43-50.

Aydin, A., \& Pearce, J. M. (1994). Prototype effects in categorization by pigeons. Journal of Experimental Psychology: Animal Behavior Processes, 20, 264-277.

Blough, D. S. (1975). Steady state data and a quantitative model of operant generalization and discrimination. Journal of Experimental Psychology: Animal Behavior Processes, 1, 3-21.

Blough, P. M. (1972). Wavelength generalization and discrimination in the pigeon. Perception \& Psychophysics, 12, 342-348.

Busey, T. A., \& Tunnicliff, J. L. (1999). Accounts of blending, distinctiveness, and typicality in the false recognition of faces. Journal of Experimental Psychology: Learning, Memory, and Cognition, 25, 1210-1235.

Cerella, J. (1979). Visual classes and natural categories in the pigeon. Journal of Experimental Psychology. Human Perception and Performance, 5, 68-77.

Cook, R. G., \& Smith, J. D. (2006). Stages of abstraction and exemplar memorization in pigeon category learning. Psychological Science, 17, 1059-1066.

Corneille, O., Goldstone, R. L., Queller, S., \& Potter, T. (2006). Asymmetries in categorization, perceptual discrimination, and visual search for reference and nonreference exemplars. Memory \& Cognition, 34, 556-567.

Corneille, O., \& Judd, C. M. (1999). Accentuation and sensitization effects in the categorization of multifaceted stimuli. Journal of Personality and Social Psychology, 77, 927-941.

Corter, J. E., \& Gluck, M. (1992). Explaining basic categories: Features predictability for information. Psychological Bulletin, 111, 291-303.

Dépy, D., Fagot, J., \& Vauclair, J. (1997). Categorization of threedimensional stimuli by humans and baboons: Search for prototype effects. Behavioural Processes, 39, 299-306.

Ghirlanda, S., \& Enquist, M. (2003). Reviews: A century of generalization. Animal Behaviour, 66, 15-36.

Gibson, E. J. (1969). Principles of perceptual learning and development. New York: Appleton-Century-Crofts.

Goldstone, R. L. (1994). Influences of categorization on perceptual discrimination. Journal of Experimental Psychology: General, $123,178-200$.

Goldstone, R. L. (1998). Perceptual learning. Annual Reviews of Psychology, 49, 585-612.

Goldstone, R. L., Steyvers, M., \& Rogosky, B. J. (2003). Conceptual interrelatedness and caricatures. Memory \& Cognition, 31, 169180.

Hanson, H. M. (1959). Effects of discrimination training on stimulus generalization. Journal of Experimental Psychology, 58, 321-334.

Harris, J. A. (2006). Elemental representations of stimuli in associative learning. Psychological Review, 113, 584-605.

Helson, H. (1964). Adaptation learning theory. New York: Harper \& Row.

Honey, R. C., \& Hall, G. (1989). Acquired equivalence and discriminative of cues. Journal of Experimental Psychology: Animal Behavior Processes, 14, 338-346.

Honey, R. C., \& Hall, G. (1991). Acquired equivalence and distinctiveness of cues using a sensory-preconditioning procedure. Quarterly Journal of Experimental Psychology, 43B, 121135.

Huber, L., \& Lenz, R. (1993). A test of the linear feature model of polymorphous concept discrimination with pigeons. Quarterly Journal of Experimental Psychology, 46B, 1-18. 
Jitsumori, M. (1993). Category discrimination of artificial polymorphous stimuli based on feature learning. Journal of Experimental Psychology: Animal Behavior Processes, 19, 244-254.

Jitsumori, M. (1994). Discrimination of artificial polymorphous categories by rhesus monkeys (Macaca mulatta). Quarterly Journal of Experimental Psychology, 47B, 371-386.

Jitsumori, M. (1996). A prototype effect and categorization of artificial polymorphous stimuli in pigeons. Journal of Experimental Psychology: Animal Behavior Processes, 22, 405-419.

Jitsumori, M., Shimada, N., \& Inoue, S. (2006). Family resemblances facilitate formation and expansion of functional equivalence classes in pigeon. Learning \&Behavior, 34, 162-175.

Jitsumori, M., \& Yoshihara, M. (1997). Categorical discrimination of human facial expressions by pigeons: A test of the linear feature model. Quarterly Journal of Experimental Psychology, 50B, 253-268.

Kruschke, J. K. (1992). ALCOVE: An exemplar-based connectionist model of category learning. Psychological Review, 99, 22-44.

Lea, S. E. G., \& Harrison, S. N. (1978). Discrimination of polymorphous stimulus sets by pigeons. Quarterly Journal of Experimental Psychology, 30, 521-537.

Lea, S. E. G., Lohmann, A., \& Ryan, C. M. E. (1993). Discrimination of five-dimensional stimuli by pigeons: Limitations of feature analysis. Quarterly Journal of Experimental Psychology, 46B, 19-42.

Lea, S. E. G., \& Ryan, C. M. E. (1983). Feature analysis of pigeons' acquisition of concept discrimination. In M. L. Commons, R. J. Herrnstein, \& A. R. Wagner (Eds.), Quantitative analyses of behavior: Acquisition processes (Vol. 4, pp. 239-253). Cambridge, MA: Ballinger.

Mackintosh, N. J. (1995). Categorization by people and pigeons: The Twenty-Second Bartlett Memorial Lecture. Quarterly Journal of Experimental Psychology, 48B, 193-214.

Makino, H., \& Jitsumori, M. (2000). Category learning and prototype effect in pigeons: A study with morphed images of human faces. Japanese Journal of Psychology, 71, 477-485.

McLaren, I. P. L., Bennett, C. H., Guttman-Nahir, T., Kim, K., \& Mackintosh, N. J. (1995). Prototype effects and peak shift in categorization. Journal of Experimental Psychology; Learning, Memory, and Cognition, 21, 662-673.

Medin, D. L., Dewey, G. I., \& Murphy, T. D. (1983). Relationships between item and category learning: Evidence that abstraction is not automatic. Journal of Experimental Psychology: Learning, Memory, and Cognition, 9, 607-625.

Nosofsky, R. M. (1986). Attention, similarity, and the identificationcategorization relationship. Journal of Experimental Psychology: General, 115, 39-57.

Nosofsky, R. M. (1991). Typicality in logically defined categories: Exemplar-similarity versus rule instantiation. Memory \& Cognition, 19, 131-150.

Nosofsky, R. M. (2000). Exemplar representation without generalization? Comment on Smith and Minda's (2000) "Thirty categori- zation results in search of a model. Journal of Experimental Psychology: Learning, Memory, and Cognition, 26, 1735-1743.

Pearce, J. M., \& Hall, G. (1980). A model for Pavlovian learning: Variations in the effectiveness of conditioned but not of unconditioned stimuli. Psychological Review, 87, 532-552.

Posner, M. I., \& Keele, S. W. (1968). On the genesis of abstract ideas. Journal of Experimental Psychology, 77, 353-363.

Reed, S. K. (1972). Pattern recognition and categorization. Cognitive Psychology, 3, 382-407.

Rilling, M. (1977). Stimulus control and inhibitory processes. In W. K. Honig \& J. E. R. Staddon (Eds.), Handbook of operant behavior (pp. 432-480). Englewood Cliffs, NJ: Prentice Hall.

Rosch, E. (1978). Principles of categorization. In E. Rosch \& B. B. Lloyd (Eds.), Cognition and categorization (pp. 27-48). Hillsdale, NJ: Erlbaum.

Rosch, E., \& Mervis, C. B. (1975). Family resemblances: Studies in the internal structure of categories. Cognitive Psychology, 7, 573-605.

Smith, J. D., Coutinho, M. V. C., \& Couchman, J. J. (2010). The learning of exclusive-or categories by monkeys (Macaca mulatta) and humans (Homo sapiens). Journal of Experimental Psychology: Animal Behavior Processes, 36, 1-10.

Smith, J. D., \& Minda, J. P. (1998). Prototypes in the mist: The early epochs of category learning. Journal of Experimental Psychology: Learning, Memory, and Cognition, 24, 1411-1436.

Smith, J. D., \& Minda, J. P. (2000). Thirty categorization results in search of a model. Journal of Experimental Psychology: Learning, Memory, and Cognition, 26, 3-27.

Soto, F. A., \& Wasserman, E. A. (2010). Error-driven learning in visual categorization and object recognition: A commonelements model. Psychological Reviews, 117, 349-381.

Spence, K. W. (1937). The differential response of animals to stimuli differing within a single dimension. Psychological Review, 44, $430-444$.

Taylor, J. R. (1995). Linguistic categorization: Prototypes in linguistic theory. Oxford: Oxford University Press.

Thomas, D. R. (1993). A model of adaptation-level effects on stimulus generalization. Psychological Review, 100, 658-673.

Thomas, D. R. (1994). The role of adaptation-level in stimulus generalization. In G. H. Bower (Ed.), The psychology of learning and motivation (Vol. 8, pp. 91-145). San Diego, CA: Academic Press.

Troje, N. F., Huber, L., Loidolt, M., Aust, U., \& Fieder, M. (1999). Catgeorical learning in pigeons: The role of texture and shape in complex static stimuli. Vision Research, 39, 353-366.

Vaughan, W., \& Greene, S. L. (1984). Pigeon visual memory capacity. Journal of Experimental Psychology: Animal Behavior Processes, 10, 256-271.

von Fersen, L., \& Lea, S. E. G. (1990). Category discrimination by pigeons using five polymorphous features. Journal of the Experimental Analysis of Behavior, 54, 69-84. 